\title{
Desempenho do Descritor Máxima Diferença na Classificação de Plantações de Eucalipto no Estado do Rio de Janeiro
}

Performance of the descriptor Max. Diff. in the classfication of Eucalyptus plantations in the Rio de Janeiro state

\author{
Debora da Paz Gomes Brandão Ferraz ${ }^{1}$ \\ Raul Sanchez Vicens ${ }^{1}$
}

Recebido em setembro de 2018.

Aprovado em março de 2019.

\begin{abstract}
RESUMO
O crescimento da área plantada de eucaliptos no Brasil vem sendo constatado por dados censitários, e a utilização de dados de sensoriamento remoto contribui para entender onde, quando e como este processo de crescimento acontece. Este trabalho tem como objetivo analisar o desempenho de indicadores espectrais de dados LANDSAT, na classificação de coberturas de eucalipto em diferentes estágios de crescimento, utilizando como área piloto o estado do Rio de Janeiro. Foram utilizados descritores de máxima diferença espectral e NDVI, extraídos de imagens do sensor OLI/LANDSAT8, numa classificação orientada à objetos (GEOBIA). A matriz de confusão utilizando pontos de validação, indicou uma exatidão total de 83,6\% e um índice kappa de 0,807. O classificador apresentou um erro de comissão de $17 \%$, principalmente com coberturas homogêneas de florestas e cultivos. O erro de omissão de $9 \%$ está distribuído principalmente em áreas de encostas, atribuindo um melhor desempenho para o classificador nas áreas planas.
\end{abstract}

PALAVRAS-CHAVE: GEOBIA, LANDSAT, Eucalipto Classificação da cobertura vegetal. 


\begin{abstract}
The growth of the eucalyptus crop area in Brazil has been verified by census data, and the use of remote sensing data helps to understand where, when and how this process happens. The work aims to analyse the performance of LANDSAT spectral features in the classification of eucalyptus land cover at different stages of growth using the state of Rio de Janeiro as the pilot area. Maximum Difference and NDVI spectral features, extracted from OLI / LANDSAT8 images, were used in an object-based classification (GEOBIA). The confusion matrix using validation points showed a total accuracy of $83.6 \%$ and a kappa index of 0.807 . The automatic classification had a commission error of $17 \%$, mainly with homogeneous forest and crop. The omission error of $9 \%$ is distributed mainly in slope areas, assigning a better performance to the classifier in the flat areas.
\end{abstract}

KEYWORDS: GEOBIA, LANDSAT, Eucalyptus, Land cover classification

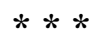

\title{
Introdução
}

No mundo inteiro, o setor florestal tem importância como fornecedor de energia ou matéria-prima para a indústria da construção civil e de transformação. No Brasil, encontram-se características singulares, já que o país está entre os principais detentores de recursos florestais abundantes, com extensas áreas de florestas tropicais (BATALHA e BUAINAIN, 2007).

A silvicultura tem um grande papel como um dos instrumentos para a obtenção de matéria-prima destinada ao atendimento de diversas demandas, que vão do pequeno agricultor à grande indústria de base florestal. Além disso, ela também tem sido utilizada para reflorestamentos destinados ao atendimento de diversas demandas ecológicas e ambientais.

Neste contexto, o reflorestamento com Eucalipto vem crescendo nas últimas décadas no país. Porém, são escassos os estudos que buscam entender como, onde e quando esse processo acontece, faltando, em alguns casos, disponibilidades de dados de área plantada. Na tentativa de preencher essas lacunas, o Sensoriamento Remoto se apresenta como um grande auxílio principalmente nas análises multitemporais, que buscam entender a dinâmica e evolução da cobertura vegetal e o uso da terra (WEIERS et al., 2004; JACKSON e JENSEN, 2005). 
Para entender melhor o comportamento da vegetação, é necessário levar em consideração que a reflexão da radiação eletromagnética das folhas dependerá da sua composição química e de estrutura interna. Já para o dossel de uma floresta, esta reflexão dependerá da quantidade de folhas e da arquitetura dele, apesar de a forma da curva de refletância de um dossel ser muito semelhante à de uma folha isolada (PAZ-PELLAT et al, 2011). Com base nesta diferença da resposta espectral dos diversos tipos de vegetação, reflorestamento, cultivos e outros, a literatura vem avançando no aprimoramento de técnicas que visam cada vez mais a uma melhor diferenciação (KAUNER et al, 2014).

Dentre as diferentes técnicas de processamento de imagens de satélite, a Análise Orientada a Objeto Geográficos (GEOBIA) vem cada vez mais se destacando na classificação de alvos complexos. Além de individualizar os objetos da cena, através da segmentação, são envolvidos no processo de classificação, além dos dados espectrais, numerosos atributos associados à forma, textura, relações contextuais e semânticas, aproximando o procedimento de classificação aos processos cognitivos humanos de interpretação de imagem, a diferencia das técnicas convencionais de classificação baseadas em pixels (CRUZ et al, 2007; BLASCHKE, 2010).

A classificação das áreas plantadas de Eucalipto através de imagens de satélite apresenta algumas dificuldades na separação da sua resposta espectral em relação a outros alvos, como por exemplo, as florestas naturais. Por este motivo, novas técnicas e abordagens vêm sendo testadas para aprimorar este tipo de classificação (FERREIRA et al, 2011; ZHOU et al, 2012; ANTUNES et al, 2014) Neste sentido, o presente trabalho tem por objetivo analisar o desempenho de um descritor de resposta espectral, com base na razão entre a máxima diferença e o brilho das bandas do visível, no intuito de classificar as coberturas de eucalipto em diferentes estágios de crescimento.

No entanto, a classificação digital de florestas plantadas apresenta certa dificuldade devido às semelhanças no comportamento espectral com as 
demais fisionomias florestais. Portanto, é necessário buscar um modelo de classificação que atenda à silvicultura, mais especificamente ao eucalipto, podendo-se analisar sua forma, sua textura e seu comportamento espectral em relação às demais classes e objetos.

Diversos trabalhos na literatura buscam contribuir para uma melhor classificação da Silvicultura. Alvares, C.A. et al (2008) compara os resultados de NDVI e EVI de áreas de Pinus no Brasil com imagens MODIS entre 2000 e 2012. Já Marsden et al (2010) faz uma relação entre a série temporal do MODIS e a estrutura, absorção de luz e produção de caules em áreas de plantios de Eucalipto.

\section{1 Área de Estudo}

A área dedicada à silvicultura no estado do Rio de Janeiro vem crescendo nos últimos anos. Segundo os dados do IBGE, correspondentes aos Censos Agropecuários e de Pesquisa de Produção de Extração Vegetal e Silvicultura (PEVS), a área plantada duplicou entre 2006 e 2016 (IBGE, 2017). Uma vez que os dados censitários coletam os totais por Unidades da Federação e no intuito de cartografar a distribuição das áreas de silvicultura no Estado do Rio de Janeiro, foi escolhida como área de estudo a superfície total do estado, a qual é coberta por um total de sete cenas de imagens Landsat (Figura 1).

Com um relevo bastante acidentado, o estado do Rio de Janeiro é marcado principalmente por três morfoestruturas: as baixadas, os maciços montanhosos e a depressão do Paraíba do Sul. Essa configuração do relevo, aliada a fatores climáticos, faz com que a vegetação do estado seja bastante diversa, contendo, por exemplo: formações pioneiras; região de floresta ombrófila densa; e região de floresta estacional semidecidual, estando esta última, presente desde o médio Paraíba até o Norte Fluminense (DANTAS et $a$ l, 2014). Esta diversidade paisagística permite, ainda, uma análise espacial 
do desempenho do classificador, uma vez que a área dedicada à silvicultura o estado, encontra-se dispersa por toda a sua superfície.

Figura 1 - Mapa de localização da área de estudo

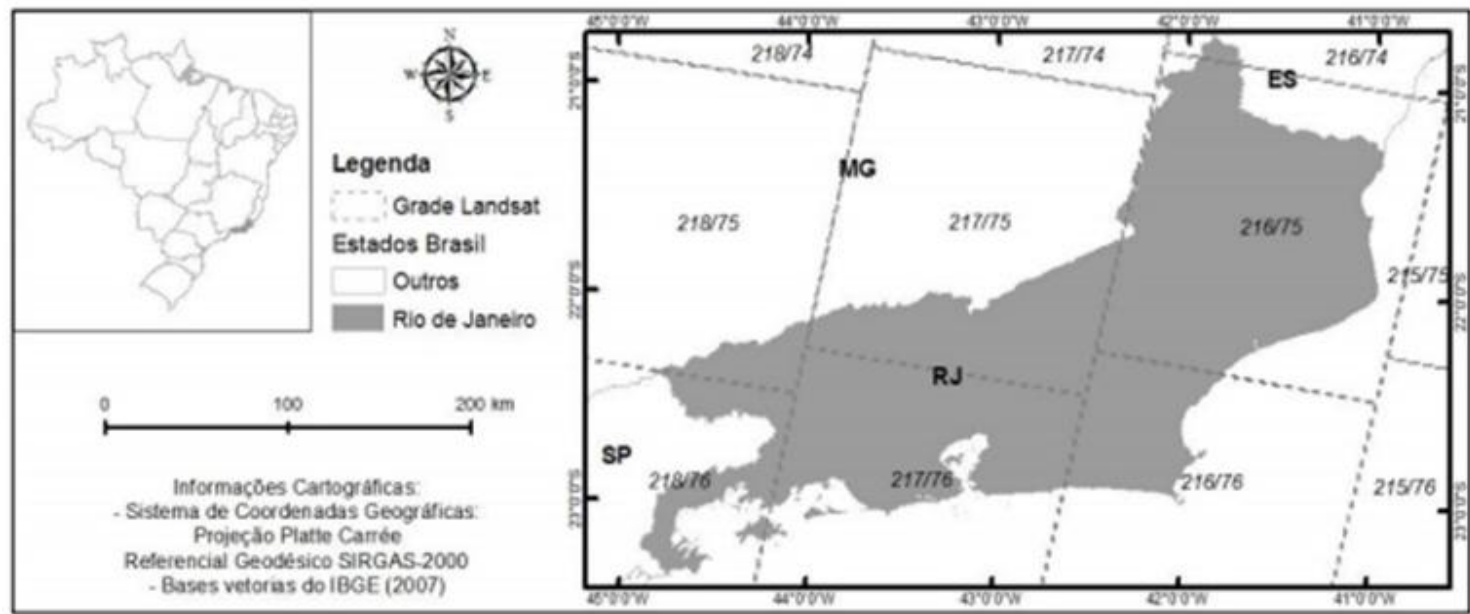

Fonte: Elaborada pelos autores

A espécie que predomina nas florestas plantadas no estado é o Eucalipto. De acordo com Mendonça-Filho (1984), no final de 1977 o eucalipto correspondia a $89 \%$ do total de 10.598 ha de povoamentos plantados no estado. Já no estudo realizado por Amorim et al (2011), foram identificadas no mapeamento, além de eucalipto e pinus, outras 10 espécies cultivadas. $\mathrm{O}$ eucalipto, entretanto, representou $97,9 \%$ da área dedicada à silvicultura, com mais de 18 mil hectares de área plantada.

\section{Material e Método}

\subsection{Imagem Landsat}

Desde seu início, em 1972, com o sensor Multispectral Scanner e posteriormente com o advento dos sensores Thematic Mapper em 1982 e Operational Land Imager (OLI) em 2013, o imageamento pelos satélites 
Landsat tem sido praticamente contínuo e soluções técnicas têm sido implementadas para maximizar a resolução espacial e espectral assim como otimizar a consistência radiométrica entre os sensores (NASA, 2016). Cabe ainda destacar esse grande avanço que representou o acesso aos dados Landsat a partir de 2008, quando o Serviço Geológico dos Estados Unidos (USGS) disponibilizou todo o acervo de imagens para download livre, com uma acurácia geométrica sem precedentes.

O Landsat 8 entrou em operação em 2013 com dois instrumentos imageadores: O Operacional Terra Imager (OLI) gera nove bandas multiespectrais com resolução espacial de 30 metros (bandas de 1 a 7 e de 9). Já a banda 8 do instrumento OLI é a pancromática e possui resolução espacial de 15 metros. O os produtos do Thermal Infrared Sensor (TIRS) equivalem às faixas do termal nas bandas 10 e 11 e têm pixels com 100 metros de resolução espacial (USGS, 2016), sendo úteis no fornecimento de temperaturas de superfície mais precisas.

Neste trabalho, foram utilizadas imagens do sensor OLI/Landsat 8 do ano de 2014. A escolha do ano teve a intenção de utilizar o ano base do projeto “Olho no Verde”, uma cooperação entre a Secretaria Estadual do Ambiente e a Universidade Federal do Rio de Janeiro, que gerou um mapeamento na escala 1:25.000 da cobertura vegetal e uso da terra (incluindo a silvicultura), utilizando imagens WorldView-2 reamostradas para 2 metros, das Regiões Hidrográficas do estado do Rio de Janeiro que contemplam o Rio Paraíba do Sul. O mapeamento de alta resolução foi, assim, utilizado na validação da classificação automática das áreas de silvicultura, utilizando imagens Landsat.

Foram escolhidas cenas do inverno de 2014, mais especificamente entre os meses de julho e agosto, para garantir uma resposta parecida dos alvos em todas as imagens e minimizar a ocorrência de nuvens sobre o estado.

As imagens foram adquiridas de forma gratuita no site da USGS (http://earthexplorer.usgs.gov/), mediante o download das 6 cenas (216/075; $216 / 076 ; 217 / 075 ; 217 / 076 ; 218 / 075 ; 218 / 075)$ que cobrem toda a área do 
estado. As cenas foram adquiridas em valores de refletância de superfície e normalizadas geometricamente.

Além das bandas espectrais, foram utilizadas como dados de entrada, as imagens sintéticas de NDVI de cada cena. Os dados foram inicialmente carregados num sistema de classificação orientado a objetos, para segmentação em diferentes níveis hierárquicos e posterior classificação supervisionada, através de amostragem e modelos probabilísticos de pertencimento às diferentes classes (Figura 2).

Figura 2. Fluxograma metodológico

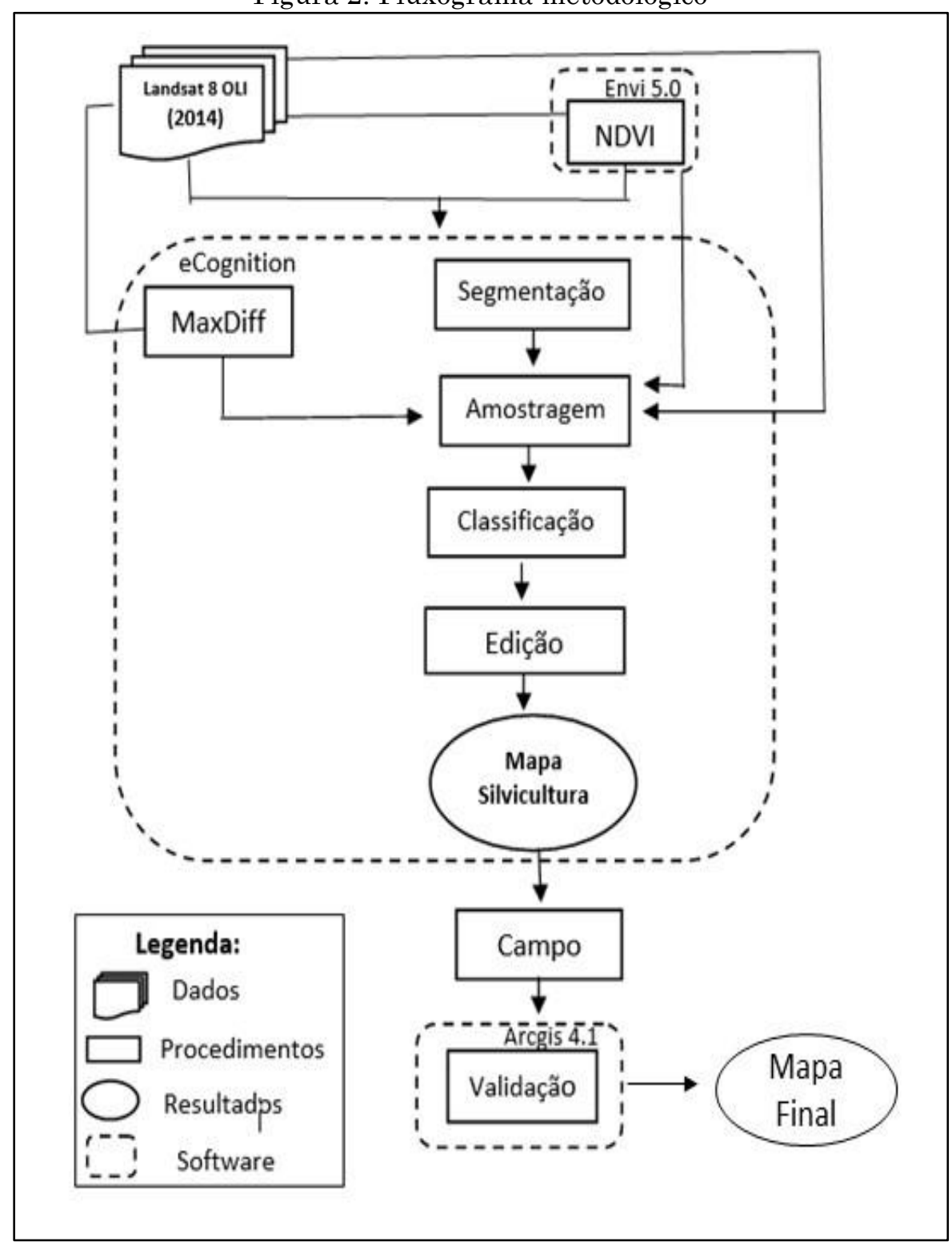

Fonte: Elaborada pelos autores 


\subsection{Classificação Orientada a Objeto}

Os testes de descritores e a classificação das áreas de silvicultura foram realizados no software eCognition 8.8. As cenas foram processadas em projetos separados para evitar erros derivados de diferenças radiométricas entre imagens adquiridas em datas diferentes. Foi utilizado o segmentador desenvolvido por Baatz e Schape (1999), multiresolution segmentation, utilizando os parâmetros de escala 50, forma 0.7 e 0.3 de compacidade. A rede semântica de classificação foi dividida em dois níveis hierárquicos: um primeiro superior de classificação das coberturas de vegetação e um nível inferior de classificação das coberturas de silvicultura (Figura 3).

Figura 3: Rede Semântica

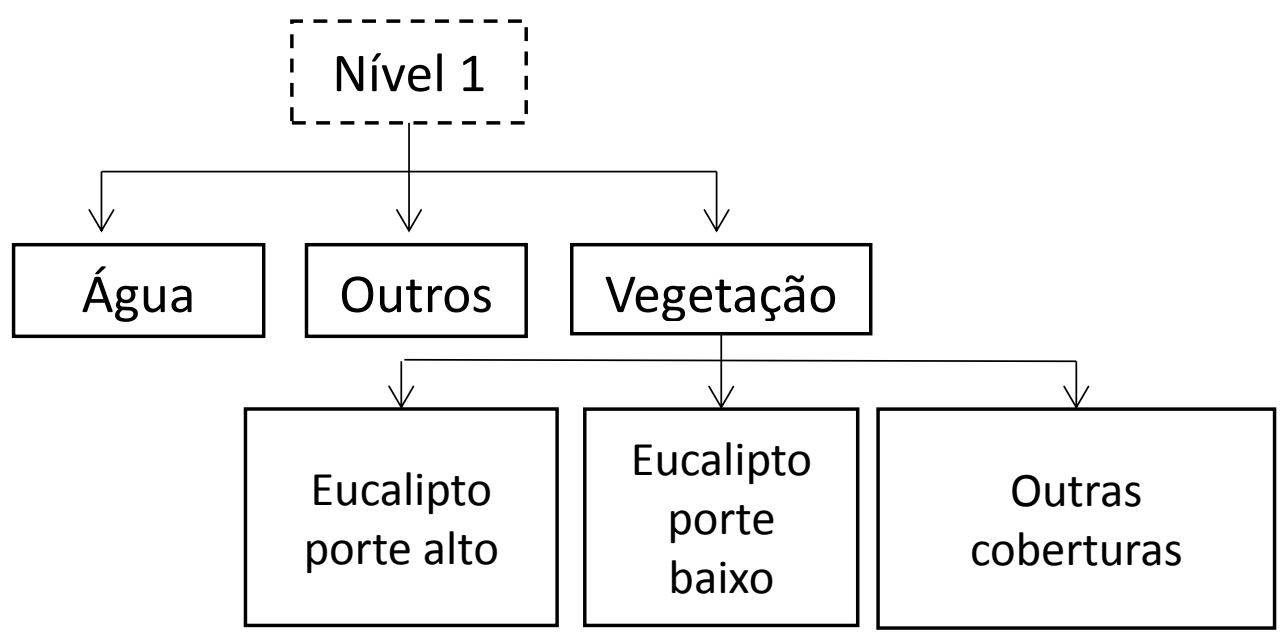

Fonte: Elaborada pelos autores

O primeiro tem o objetivo de separar a vegetação das outras classes, sendo elas: água e outras coberturas (áreas desprovidas de vegetação como solos expostos, áreas construídas, nuvens e sombras). Já o segundo, classificado a partir do resultado e da edição do anterior, tinha o objetivo de separar a plantação de alto e baixo porte. 
No primeiro nível, para separar a classe água foi utilizado o valor médio de refletância no Infravermelho Próximo, e uma função probabilística (fuzzy) para classificar objetos com valores inferiores a 20. Para as demais classes, foi utilizada a coleta de amostras, tendo uma média de 5 a 10 amostras por classe e variando de acordo com a representatividade das mesmas dentro das cenas. Para as coberturas com vegetação, foi utilizado como descritor o NDVI e um limiar de classificação de 0,5.

No segundo nível hierárquico de classificação, foram utilizados dois descritores: i) Max.Diff., no intuito de separar a silvicultura de porte alto das florestas e ii) NDVI para separar as silviculturas de baixo porte das pastagens.

$\mathrm{O}$ índice Max. Diff. (Máxima diferença do Brilho) está relacionado à maior diferença encontrada entre as refletâncias médias de cada objeto, representado como:

$$
\max \left|b_{i}(k)-b_{j}(k)\right|
$$

Onde $b_{i}(k)$ e $b_{j}(k)$ são os valores médios do objeto $k$ nas bandas $\mathrm{i}$ e $\mathrm{j}$ respectivamente. $O$ índice é então calculado como a razão da maior diferença de refletâncias entre as bandas do visível e o brilho total:

$$
\text { Max.Diff }=\frac{\max \left|b_{i}(k)-b_{j}(k)\right|}{\bar{b}(k)}
$$

O índice varia de 0 a 1 e é gerado de forma automática pelo software eCognition (DORAVANA et al, 2011; LI et al, 2011; eCognition Definiens, 2014). O Brilho também é gerado de forma automática e consiste no somatório dos brilhos das bandas que correspondem ao espectro do visível.

O NDVI (Índice de Vegetação por Diferença Normalizada), proposto por Rouse et al. (1973), é um indicador sensível ao acúmulo de biomassa verde e cujos valores variam no intervalo de -1 a 1 (JENSEN, 2009; BORATTO, I. e GOMIDE, R., 2013).

O Quadro 1 apresenta os descritores utilizados para a modelagem das classes. Os modelos utilizados são, em grande maioria, de lógica fuzzy, 
relacionados à probabilidade de pertencimento a uma determinada classe. Foi utilizado um modelo booleano por exclusão (not) para as classes que não era eucalipto, ou seja, que não eram o foco do mapeamento.

\begin{tabular}{|c|l|}
\hline \multicolumn{2}{|c|}{ Quadro 1- Descritores utilizados na classificação } \\
\hline \multicolumn{2}{|c|}{ Primeiro Nível } \\
\hline Água & Infravermelho Próximo $(<20)$ \\
\hline Outros & $\begin{array}{l}\text { (not) vegetação } \\
(\text { not) água }\end{array}$ \\
\hline Vegetação & $\begin{array}{l}\text { NDVI (>0.5) } \\
\text { (not água) }\end{array}$ \\
\hline Eucalipto & \multicolumn{1}{|c|}{ Segundo Nível } \\
\hline Porte Alto & Max. Diff. (Intervalo entre 0.55 a 0.85) \\
\hline Eucalipto & NDVI (Intervalo entre -0.075 a 0.17) \\
\hline Porte Baixo & $\begin{array}{l}\text { (not) eucalipto porte alto } \\
(\text { not) eucalipto porte baixo }\end{array}$ \\
\hline Outras coberturas vegetais
\end{tabular}

Fonte: Elaborado pelos autores

Após a classificação automática foi realizada uma edição manual das classes do primeiro nível, seguida pela segmentação do segundo nível de classificação, onde as novas classes herdam as informações contidas no nível superior. Neste nível de sub-objetos foram modeladas as duas fisionomias de eucalipto, segundo o porte da plantação, separando-as das restantes coberturas vegetais.

\subsection{Análise da Acurácia}

A acurácia de mapeamentos de dados globais é fundamental tanto para estabelecer a exatidão dos produtos para a comunidade científica. E diante disso, diversas técnicas de análises vêm sendo desenvolvidas, principalmente referentes a classificação a partir de imagens digitais (COHEN e JUSTICE, 1999). 
No presente trabalho foi utilizada a matriz de confusão ou erro. Como uma tabulação cruzada simples, a matriz de confusão constitui a base sobre que descreve a precisão da classificação e caracteriza os erros, podendo ajudar a refinar a classificação ou estimativa derivada dela (CONGALTON et al., 1983; CONGALTON, 1988; CONGALTON e GREEN, 1993; STEHMAN, 1997; FOODY, 2002; VAN OORT, 2007).

Com a finalidade de verificar a acurácia da classificação deste trabalho, foi realizada uma avaliação através do índice Kappa, utilizando como verdade terrestre pontos coletados no campo e mapas de referências com uma maior escala de detalhes. Segundo Antunes e Lingnau (2004) o coeficiente de acurácia Kappa para classes individuais, parece ser o mais indicado na avaliação da classificação de imagens de sensoriamento remoto, por levar em conta os erros comissão e omissão (STEHMAN, 1996; GALFORD et al., 2008; FORKEL et al., 2013; ULLMANN et al., 2014; ABADE et al., 2015; GAO et al., 2015; XU et al., 2015).

Sendo assim, foram gerados de forma aleatório no software Arcgis 10.1, 420 pontos. A verificação dos pontos foi feita a partir de duas verdades: a primeira, tendo como verdade terrestre os resultados do Projeto "Olho no Verde”. Já a segunda, foi a verificação também de pontos aleatórios em campo das áreas não contempladas no projeto anterior.

\section{Resultados e Discussões}

Há uma precariedade de dados de área plantada de silvicultura no estado do Rio de Janeiro ao longo do tempo. Não existe uma continuidade de aquisição desses dados e eles apenas são encontrados a níveis estaduais, não estando disponível essa informação em relação aos municípios. A área plantada em todo o estado é informada apenas nos Censos Agropecuários (1975, 1980, 1985, 1996 e 2006) e pela pesquisa PEVS (Produção da Extração Vegetal e Silvicultura), que passou a coletar os dados anualmente, por municípios, a partir de 2014. 
$\mathrm{Na}$ tentativa de suprir tal falta de dados temporais, o Sensoriamento Remoto se apresenta como uma excelente ferramenta de obtenção dessas informações vista a disponibilidade de séries temporais de imagens e modelos de classificação que permitem a reconstrução da trajetória evolutiva recente das áreas de silvicultura.

As áreas de silvicultura no estado do Rio de Janeiro, e em particular as áreas de eucalipto, apresentam diferentes estágios de crescimento segundo a forma como são manejados. As fisionomias de porte alto ou arbóreo, correspondem aos estados avançados, próximos ao corte e que apresentam um alto grau de confusão com as florestas naturais ou com as florestas secundárias em estado avançado de recuperação. Para tentar distinguir entre ambas fisionomias, foi escolhido o descritor Max. Diff.

Com o objetivo de testar e melhor compreender o classificador Max. Diff, foram coletadas 50 amostras correspondentes à geo-objetos classificados como coberturas de Eucalipto de porte alto e 50 amostras de floresta. Utilizando os valores médios de Max. Diff., nas 100 amostras, constatou-se que o descritor consegue separar bem as duas classes, pois o eucalipto se concentra em um grupo de valores que vai de 0.55 a 0.85 , enquanto que a floresta apresentou valores entre 0.85 e 1. Em resumo, a floresta apresenta, em geral, maiores valores de Max. Diff. que o eucalipto de grande porte (Figura 4).

Figura 4: Janela do Sample Editor do eCognition, mostrando os histogramas das amostras de eucalipto de porte alto e floresta no descritor de Máxima diferença.

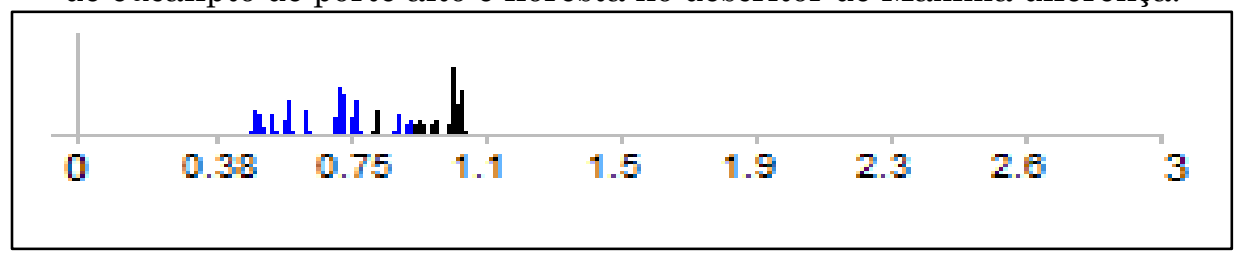

Fonte: Elaborado pelos autores

Uma análise detalhada das variáveis que compõem a razão da máxima diferença espectral (Gráfico 1), indica um comportamento similar do eucalipto de porte alto e da floresta, principalmente quanto a maior amplitude entre 
bandas no visível (ordenadas). No entanto, a floresta apresenta maiores valores de brilho (abscissas) que resulta em menores valores de Max Diff.

Gráfico 1 - Valores de maior amplitude entre bandas do visível e brilho total em amostras de Eucalipto e Floresta

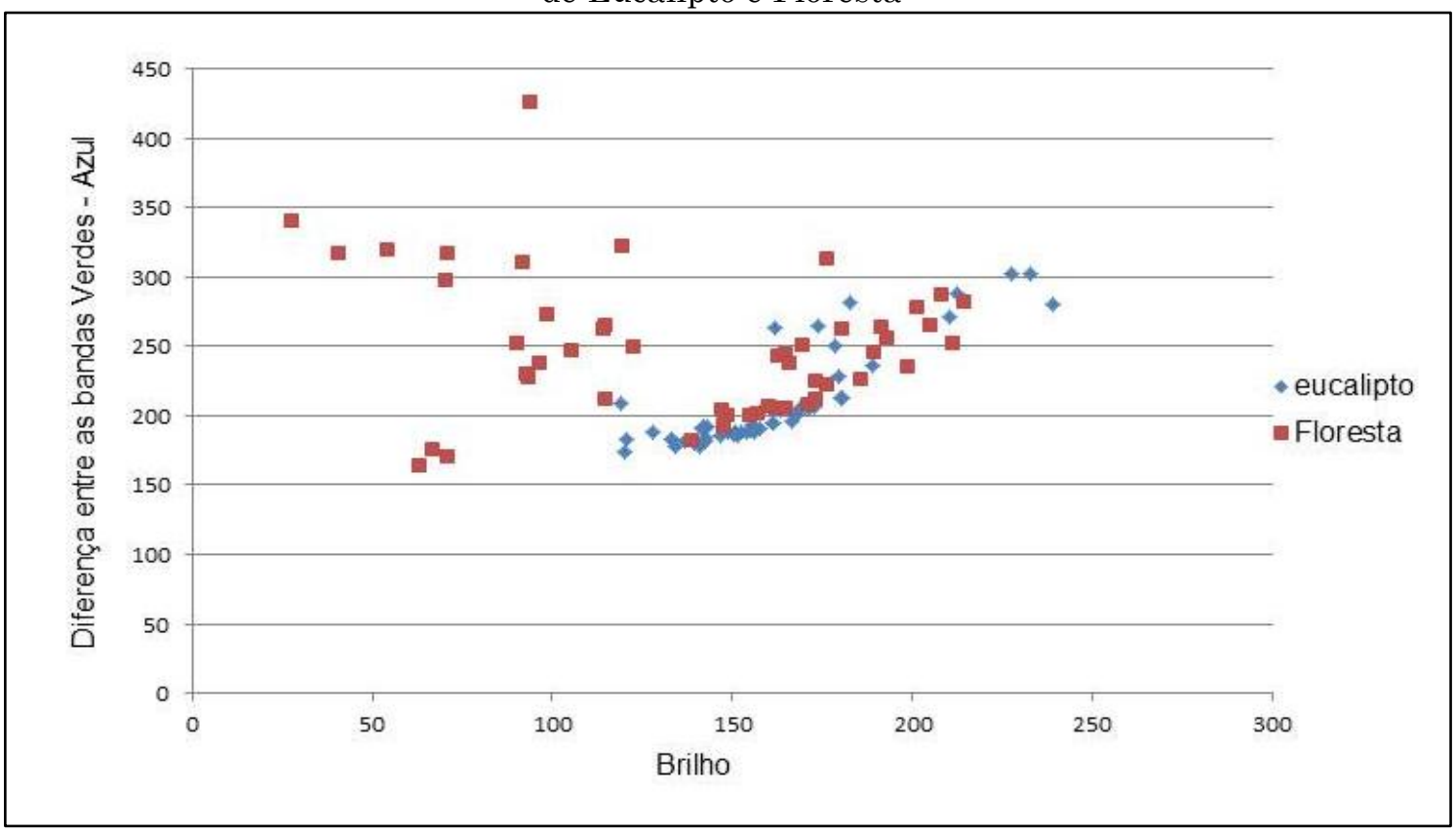

Fonte: Elaborado pelos autores

Em geral, floresta apresenta maiores valores de Max Diff. que o Eucalipto, porém há amostras com valores muito próximos o qual pode ser explicado por determinadas condições, como a declividade das encostas ou o porte e a composição da própria vegetação.

O eucalipto de porte baixo tende a misturar-se com as coberturas de pasto, principalmente na parte do visível do espectro. O melhor descritor encontrado para separar ambas as classes foi o NDVI. Após a definição dos melhores parâmetros ou limiares para cada descritor, foi feita a classificação automática utilizando modelos probabilísticos (fuzzy) e os resultados submetidos à validação.

A validação do mapeamento se baseou em duas verdades (Figura 5). A primeira a partir dos resultados do Projeto "Olho No Verde", que utilizou imagens WorldView-2. Sendo assim, para as áreas do mapeamento que correspondem às Regiões Hidrográficas do Médio Paraíba do Sul (RH-III), 
Piabanha (RH-IV), Rio Dois Rios (RH-VII), e a parte Sul da Região Hidrográfica do Baixo Paraíba do Sul e Itabapoana (IX) do estado do Rio de Janeiro, foi feita uma comparação (Intersect), no software Arcgis 10.4, dos polígonos das áreas classificadas pelo projeto como de silvicultura, e também das geradas automaticamente pelos classificadores.

Figura 5 - Pontos de Controle

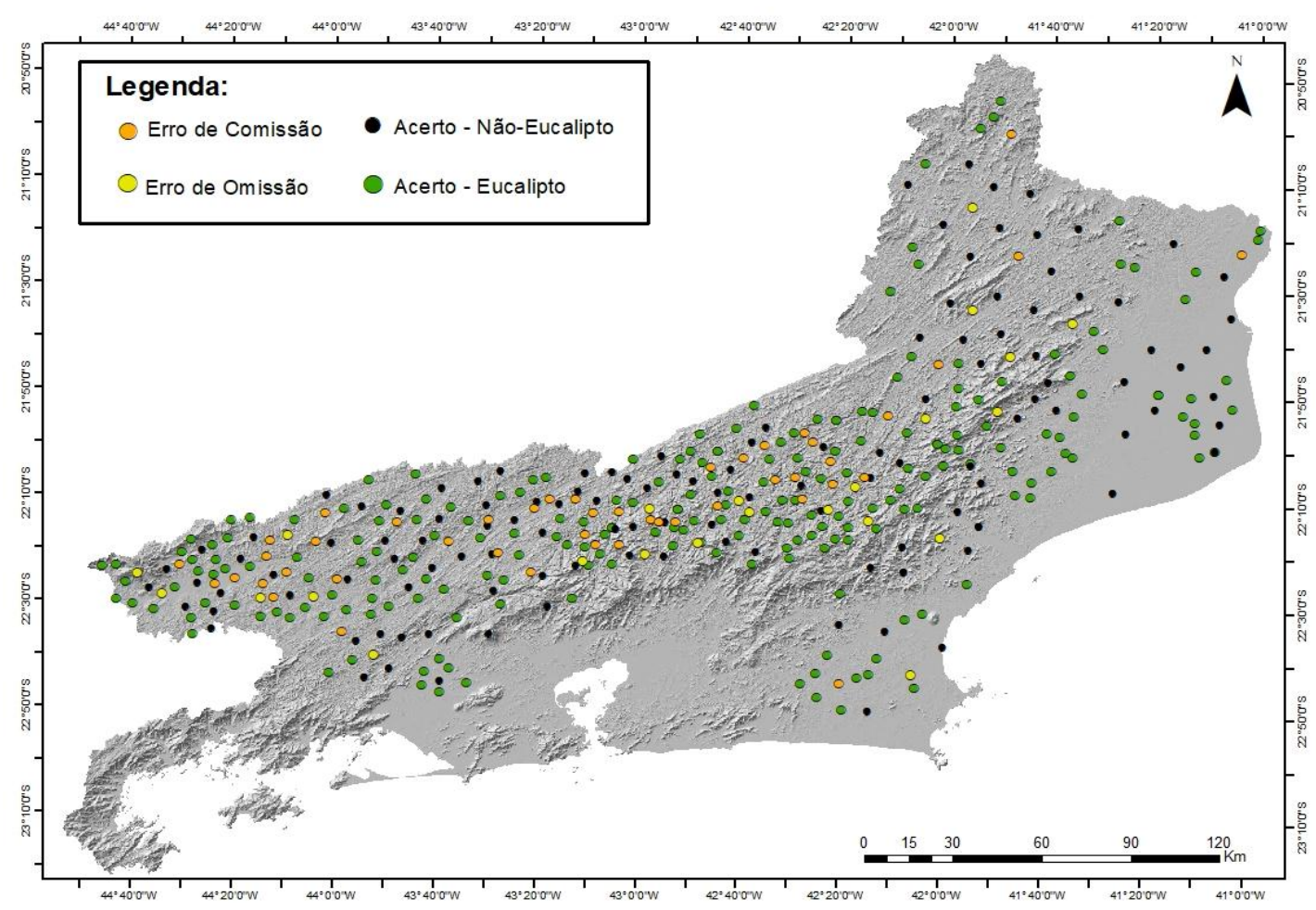

Fonte: Elaborada pelos autores

Por se tratarem de mapeamentos com escala de detalhes diferentes, foram excluídas do projeto as áreas de menos de dois hectares. Isso foi feito pois, na classificação realizada a partir das imagens Landsat, era difícil distinguir áreas menores do que essa. Foram validados, no total, 162 pontos de silvicultura e 85 de não-silvicultura.

Para o restante do mapa a validação foi feita a partir de pontos de controles gerados de forma aleatória no software Arcgis 10.4, os quais foram visitados em campo. Para cada uma das áreas correspondentes às Regiões Hidrográficas do Guandu (RH-II), Baía de Guanabara (RH-V), Lagos São João 
(RH-VI), Macaé e das Ostras (RH-VIII) e a parte Norte da Região Hidrográfica do Baixo Paraíba do Sul e Itabapoana (RH-IX), foram geradas em torno de 30 pontos de controle.

De acordo com Congalton e Green (2009), o número mínimo necessário é de 50 amostras por classe, para mapas cobrindo menos de um milhão de acres e com menos de 12 classes. Porém, por dificuldade de acesso, foram visitados no campo 108 pontos de silvicultura e 65 de não-silvicultura.

A matriz de confusão (Tabela 1) com um total de 270 pontos para a classe de silvicultura e 150 para a de não-silvicultura, indicou exatidão total de $83,5 \%$ e um índice kappa de 0,807. A tabela mostra também os erros de omissão e de comissão, os primeiros se referindo às amostras que não foram classificadas de acordo com as classes às quais se foi dito que pertenciam. Já os outros estão relacionados às amostras de referência e são classificadas as classes a que elas não pertencem. (FRANCISCO e ALMEIDA, 2012).

\begin{tabular}{|c|c|c|c|c|}
\hline & & \multicolumn{2}{|c|}{ Classificação } & \multirow{2}{*}{ Total } \\
\hline & & Eucalipto & Outros & \\
\hline \multirow{2}{*}{ Referência } & Eucalipto & 224 & 23 & 247 \\
\hline & Outros & 46 & 127 & 173 \\
\hline \multicolumn{2}{|c|}{ Total } & 270 & 150 & 420 \\
\hline \multicolumn{2}{|c|}{ Erro de Comissão } & $17,0 \%$ & $15,3 \%$ & \\
\hline \multicolumn{2}{|c|}{ Erro de omissão } & $9,3 \%$ & $26,6 \%$ & \\
\hline \multicolumn{2}{|c|}{ Exatidão total } & \multicolumn{3}{|c|}{$83,5 \%$} \\
\hline \multicolumn{2}{|c|}{ Kappa } & \multicolumn{3}{|c|}{0,807} \\
\hline
\end{tabular}

Os erros de comissão não apresentam um padrão de ocorrência ou distribuição espacial, confundindo as áreas de eucalipto com áreas de florestas mais homogêneas, com áreas de cultivo (por exemplo, de coco e de banana) e até com área de mangue.

As áreas de silviculturas, que não foram classificadas corretamente (erros de omissão) predominaram nas áreas de encostas, com um padrão de comportamento bem definido, o que mostra que o classificador tem um melhor desempenho em áreas planas. 
Os erros representam $16,42 \%$ do total de amostras utilizadas na validação, associados principalmente a pequenas áreas, localizadas em encostas (Figura 5). Os polígonos não ultrapassam as 20 ha e em sua maioria possuem uma área entre 2 e 6 hectares.

A distribuição espacial das áreas de eucalipto na data do mapeamento (2014), obtida na classificação, indicou que, apesar de não muito expressiva, elas ocupam cerca de 30.000 ha da superfície de todo o estado, sendo os municípios com maiores áreas Resende, Nova Friburgo, Vassouras, Duas Barras e Paraíba do Sul (Figura 6). Foram consideras as áreas planadas maiores que 2 ha devido a resolução das imagens de satélite utilizadas no mapeamento. Resultados da pesquisa Produção de Extração Vegetal e Silvicultura, divulgada pelo IBGE, indicaram uma área plantada de Silvicultura no estado do Rio de Janeiro, para o mesmo ano, de 37.609 ha. A diferença entre os valores pode estar associada à metodologia de levantamento dos dados, pois a metodologia utilizada pelo IBGE consiste na coleta de informações mediante aplicação de questionários. O agente coleta as informações consultando estabelecimentos agropecuários, indústrias e outros órgãos atuantes no setor.

Apesar das diferentes metodologias utilizadas, o mapeamento realizado por Amorim e colaboradores em 2011, já mostrava como a maior área de concentração de silvicultura do estado no Médio Paraíba, distribuídas em pequenos e médios fragmentos de área plantada. 
Figura 6 - Distribuição espacial das áreas de Eucalipto no estado do Rio de Janeiro

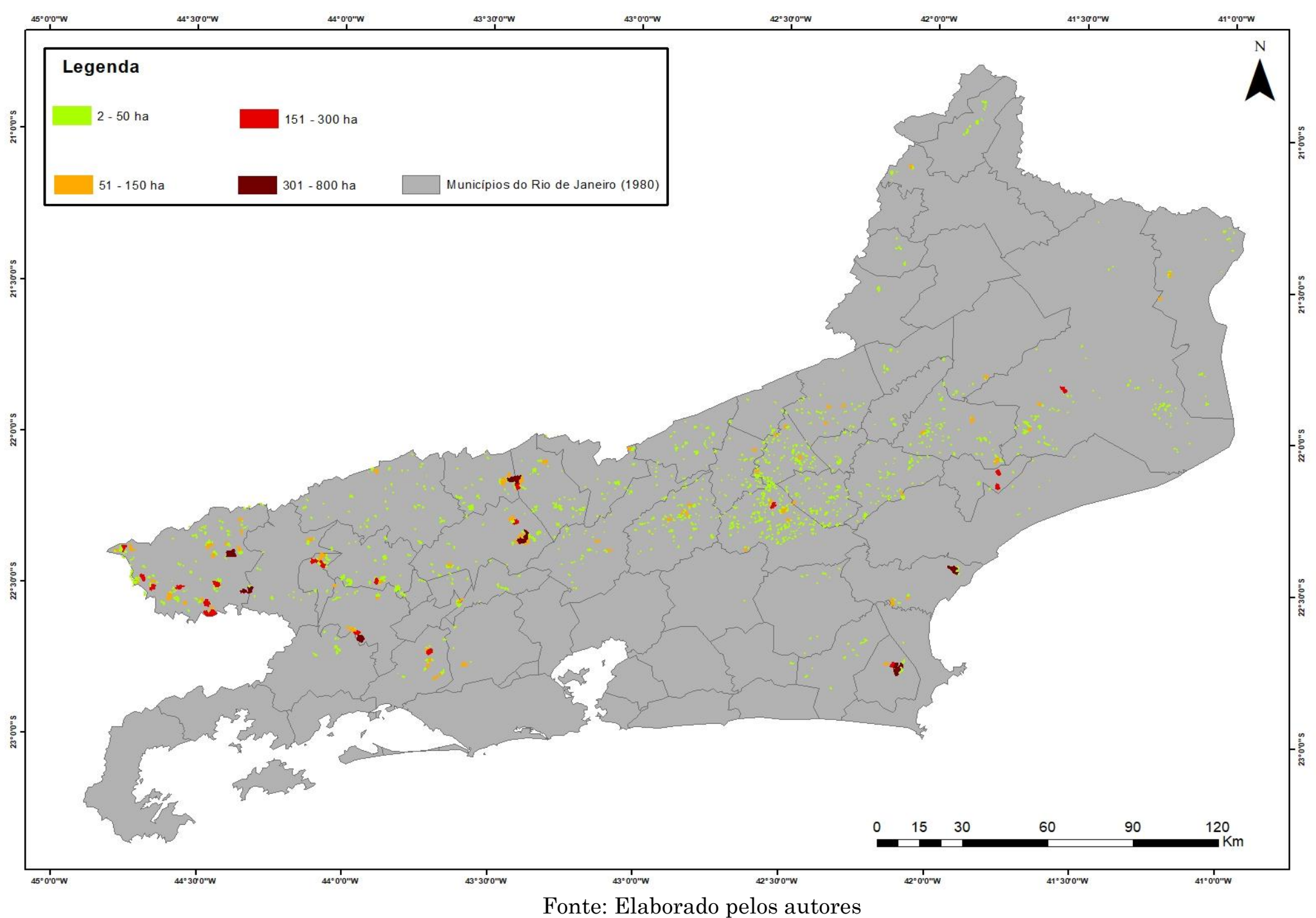


Diferente do que acontece em outros estados, onde há grandes áreas contínuas de silvicultura, no estado do Rio de Janeiro a área plantada de eucaliptos é muito fragmentada, em sua grande maioria de pequenos fragmentos (Gráfico 2). O padrão de distribuição espacial fragmentado e disperso reflete o controle exercido pelo relevo sobre a silvicultura, restringindo a continuidade e, por tanto, o tamanho das áreas plantadas.

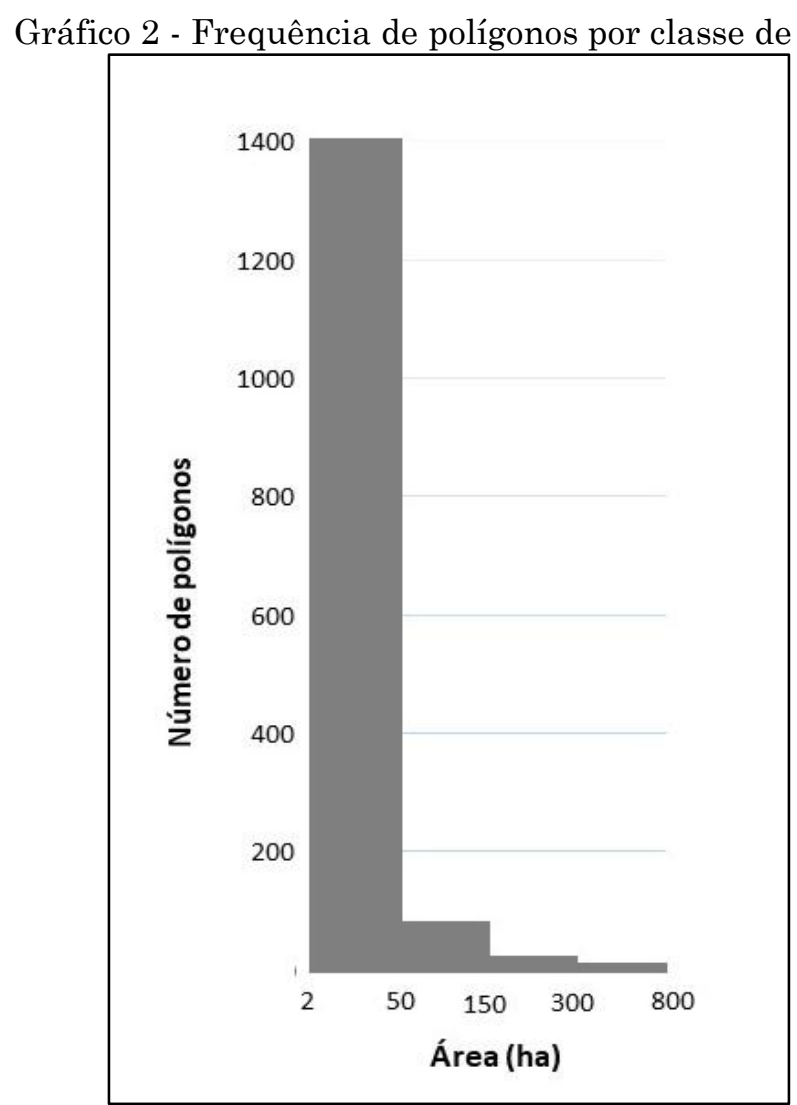

Fonte: Elaborado pelos autores

\section{Considerações Finais}

O estudo testou o desempenho de um descritor de resposta espectral, com base na razão entre a máxima diferença e o brilho das bandas do visível, mapeando as áreas plantadas de eucalipto no estado do Rio de Janeiro em 2014, com o uso de imagens Landsat/OLI. O método de deteç̧ão proposto demonstrou adequado para o objeto de estudo e para a escala a nível estadual. 
Com um Kappa de 0,807, a classificação teve um desempenho favorável. Os principais erros na classificação aconteceram em áreas de encosta, devido ao sombreamento do relevo e à existência de alguns tipos de cultivos com os quais o classificador apresentou certo grau de confusão. $\mathrm{O}$ mesmo aconteceu nas bordas de áreas de florestas, devido a escala utilizada. No entanto, o desempenho do classificador Max. Diff. mostrou-se promissor da diferenciação de coberturas de eucalipto em estágio avançado

O mapeamento obtido para dados de 2014 permitiu constatar ainda que a área dedicada a silvicultura cresceu no estado do Rio de Janeiro em relação a 2009 (AMORIM et al, 2012), mantendo um padrão fragmentado em pequenas áreas.

\section{Agradecimentos}

À Capes à Universidade Federal Fluminense e ao Programa de PósGraduação em Geografia. Ao Laboratório de Geografia Física (Lagef) por toda infraestrutura disponível.

\section{Referências}

ABADE, N. A.; DE CARVAlHO, O. A.; GUIMARÃES, R. F.; DE OLIVEIRA, S. N. Comparative analysis of MODIS time-series classification using support vector machines and methods based upon distance and similarity measures in the brazilian cerrado-caatinga boundary. Remote Sensing, vol. 7, n. 9, 2015. pp. $12160-12191$

ABRAF - Associação Brasileira de Produtores de Florestas Plantadas. Anuário Estatístico Abraf 2013 - Ano-Base 2012. Brasília, 2013.

ABREU, M.B. Análise Espaço temporal da Cobertura e Uso da Terra no Estado do Rio de Janeiro de 1994 até 2007. Dissertação de Mestrado em Geografia, PPGG, UFRJ. Rio de Janeiro, RJ, 2010.

ALVARES, C. A.; MUNHOZ, J. S. B.; STAPE, J. L.; STAHL, J.; ANDRADE, L.; SANTOS, G.; LIMA, R.; COUTINHO, R. T.; HOINACKI, R.; FOX, T. 
Caracterização da dinâmica dos índices de vegetação NDVI e EVI em plantações de Pinus do Brasil. Anais do XVI Simpósio Brasileiro de Sensoriamento Remoto, Foz do Iguaçu, Brasil, 2013. pp. 6917-6922.

AMORIM, H. B., FRANCELINO, M. R., SALAMENE, S., PEDREIRA, L. O. L., ASSUMPÇÃO FILHO, L. I. D., CAPITANO, R. C., e MOURA, T. A. Estimativa da área ocupada por reflorestamentos no Estado do Rio de Janeiro. Cerne, vol. 18, n.1, 2012. pp. 27-32.

ANTUNES, R. R.; BIAS, E.; BRITES, R. S.; COSTA, G. A. O. P. Desenvolvimento de técnica para monitoramento do cadastro urbano baseado na classificação orientada a objetos. Estudo de caso: Município de Goianésia, Goiás. Revista Brasileira de Cartografia, vol. 67, n. 2, 2014. pp.357-372.

BAATZ, M.; SCHAPE, A. Multiresolution segmentation: an optimization approach for high quality multi-scale image segmentation. AGIT-Symposium Salzburg, Karlsruhe. International Journal of Remote Sensing, vol.10, n.06, 1999. pp. 989-1003.

BLASCHAKE, T. Object based image analysis for remote sensing. ISPRS Journal of Photogrammetry e Remote Sensing, vol. 65, 2010. pp. 2-16

BORATTO, I. M. P.; GOMIDE, R. L. Aplicação dos índices de vegetação NDVI, SAVI e IAF na caracterização da cobertura vegetativa da região Norte de Minas Gerais. Anais do XVI Simpósio Brasileiro de Sensoriamento Remoto, Foz do Iguaçu, Brasil, 2013. pp. 7345-7352.

BUAINAIM, A.M.; BATALHA, M.O. Cadeia produtiva de madeira. Ministério da Agricultura, Pecuária e Abastecimento, Secretaria de Política Agrícola, Instituto Interamericano de Cooperação para a Agricultura, 2007. 84p.

COHEN, W. B.; JUSTICE, C. O. Validating MODIS terrestrial ecology products: Linking in situ and satellite measurements. Remote Sensing of Environment, vol. 70, n. 1, 1999. pp.1-3

CONGALTON, R. G. Using spatial autocorrelation analysis to explore the errors in maps generated from remotely sensed data. Photogrammetric engineering and remote sensing, vol. 54, n. 5, 1988a. pp. 587-592

CONGALTON, R. G.; GREEN, K. A practical look at the sources of confusion in error matrix generation. Photogrammetric Engineering e Remote Sensing, vol. 59, n. 5, 1993. pp. 641-644 
CONGALTON, R. G.; ODERWALD, R. G.; MEAD, R. A. Assessing Landsat Classification Accuracy Using Discrete Multivariate Analysis Statistical Techniques. Photogrammetric Engineering and Remote Sensing, vol. 49, n. 12 , 1983. pp. $1671-1678$

CRUZ, C.B.M.; VICENS, R. S.; ROSÁRIO, L. S.; ABREU, M. B.; ALMEIDA, P. M. M.; CRONEMBERGER, F.M. Classificação orientada a objetos na geração do mapa de uso e cobertura do estado do Rio de Janeiro. Anais do XIV Simpósio Brasileiro de Sensoriamento Remoto, Natal, Brasil, 2009. pp.7789-7796

DRONAVA, I.; GONG, P.; WANG, L. Object-based analysis and change detection of major wetland cover types and their classification uncertainty during the low water period at Poyang Lake, China. Remote Sensing of Environment, vol 115, n 12, 2011. pp.3220-3236.

ECOGNTION DEVELOPER. Reference Book. Trimble. Version 9.1.3. Disponível em: <www.eCognition.com>, acesso em Dezembro de 2015.

FERREIRA, J. H. D., DE OLIVEIRA, M. A., VIERO, G. P., FERREIRA, R. J. D., PEREIRA, P. S.; DE SOUZA FILHO, E. E. Diferenciação da Silvicultura de Eucalipto e Pinus, na região da bacia hidrográfica do Tibagi. Revista Brasileira de Cartografia, vol. 63, n. 2, 2011. pp. 203-210.

FRANCISCO, C.; ALMEIDA, C. Interpretação de imagens orbitais por meio de sistema especialista para o mapeamento de cobertura da terra em região montanhosa. Sociedade e Natureza, vol. 24, n. 2, 2012. pp. 283-302.

FOODY, G. M. Status of land cover classification accuracy assessment. Remote Sensing of Environment, vol. 80, n. 1, 2002. pp. 185-201.

FORKEL, M.; CARVALHAIS, N.; VERBESSELT, J.; MAHECHA, M. D.; NEIGH, C. S. R.; REICHSTEIN, M. Trend Change detection in NDVI time series: Effects of inter-annual variability and methodology. Remote Sensing, vol. 5, n. 5, 2013. pp. 2113-2144

GALFORD, G. L.; MUSTARD, J. F.; MELILlO, J.; GENDRIN, A.; CERRI, C. C.; CERRI, C. E. P. Wavelet analysis of MODIS time series to detect expansion and intensification of row-crop agriculture in Brazil. Remote Sensing of Environment, vol. 112, n. 2, 2008. pp. 576-587

GAO, T.; ZHU, J.; ZHENG, X.; SHANG, G.; HUANG, L.; WU, S. Mapping spatial distribution of larch plantations from multi-seasonal landsat-8 OLI imagery 
and multi-scale textures using random forests. Remote Sensing, vol. 7, n. 2, 2015. pp. 1702-1720

INSTITUTO BRASILEIRO DE GEOGRAFIA E ESTATÍSTICA (IBGE) - Produção de Extração Vegetal e Silvicultura (PEVS). In: http://www.sidra.ibge.gov.br/bda/pesquisas/pevs/. Acessado em novembro de 2016.

JACKSON, M. W.; JENSEN, J. R. An Evolution of Remote Sensing derived Landscape Ecology Metrics for Resevoir Shoreline Enviromental Monitoring. Photogrammetric Engineering e Remote Sensing, vol. 71, n. 12, 2005. pp.1387-1397.

JENSEN, J. R. Sensoriamento remoto do ambiente: uma perspectiva em recursos naturais. São José dos Campos, SP: Parêntese, 2009.

KNAUER, K.; GESSNER, U.; DECH, S; KUENZER, C. Remote sensing of vegetation dynamics in West Africa. International Journal of Remote Sensing, vol. 35, n. 17, 2014. pp.6357-6396.

LI, W.; OUYANG, Z.; ZHOU, W.; CHEN, Q. 2011. Effects of spatial resolution of remotely sensed data on estimating urban impervious surfaces. Journal of Environmental Sciences, vol. 23, n. 8, 2011. pp.1375-1383.

MARDSEN, C.; LE MAIRE, G.; STAPE, J.L.; Lo SEEN, D.; ROUPSARD, O.; CABRAL, O.; EPRON, D.; LIMA, A.M.N.; NOUVELLON, Y. Relating MODIS vegetation index time-series with structure, light absorption and stem production of fast-growing Eucalyptus plantations. Forest Ecology and Management, vol. 259, n. 9, 2010. pp. 1741- 1753

MENDONÇA-FILHO, W. F. (Coord.). Inventário florestal nacional: reflorestamento: Rio de Janeiro e Espírito Santo. Brasília: IBDF, 1984.

NATIONAL AERONAUTICS AND SPACE ADMINISTRATION (NASA). Landsat

Science. Disponível: < https://landsat.gsfc.nasa.gov/landsat-8/> Acessado em: 08 de outubro de 2016.

PAZ-PELLAT, M., REYES, R., MEDRANO, E. Diseño de índices espectrales de la vegetación usando curvas iso-suelo. Agrociencia vol. 45, 2011. pp.121-134.

ROUSE, J.W.; HAAS, R.H.; SCHELL, J.A.; DEERING, D.W. Monitoring vegetation systems in the Great Plains with ERTS. Proceedings of the 3rd ERTS Symposium, NASA SP-351 I, 1973. pp. 309-317. 
STEHMAN, S. V. Selecting and interpreting measures of thematic classification accuracy. Remote Sensing of Environment, vol. 62, n. 1, 1997. pp. 77-89

STEHMAN, S. V. Estimating the Kappa coefficient and its variance under stratified random sampling. Photogrammetric Engineering e Remote Sensing, vol. 62, n. 4, 1996. pp. 401-407

ULLMANN, T.; SCHMITT, A.; ROTH, A.; DUFFE, J.; DECH, S.; HUBBERTEN, H. W.; BAUMHAUER, R. Land cover characterization and classification of arctic tundra environments by means of polarized synthetic Aperture X- and C-band radar (PolSAR) and Landsat 8 multispectral imagery - Richards Island, Canada. Remote Sensing, vol. 6, n. 9, 2014. pp. 8565-8593.

UNITED SERVICE GEOLOGICAL SURVEY (USGS). Earth Explorer. Disponível em: < https://earthexplorer.usgs.gov/> Acessado em: 16 de outubro de 2016.

VAN OORT, P. A. J. Interpreting the change detection error matrix. Remote Sensing of Environment, vol. 108, n. 1, 2007. pp. 1-8.

WEIERS, S., BOCK, M., Wissen, M., ROSSNER, G., 2004, Mapping and indicator approaches for the assessment of habitats at different scales using remote sensing and GIS methods. Landscape and Urban Planning, 67, pp. 43-65.

XU, Y.; SHI, J.; DU, J. An improved endmember selection method based on vector length for MODIS reflectance channels. Remote Sensing, vol. 7, n. 5, 2015. pp. 6280-6295.

ZHOU, J., PROISY, C., DESCOMBES, X., LE MAIRE, G., NOUVELLON, Y., VIENNOIS, G., ZERUBIA, J., COUTERON, P. Mapping local density of young eucalyptus plantations by individual tree detection in high spatial resolution satellite images. Forest Ecology and Management, vol. 301, 2013. pp.129141. 